\title{
O CONSUMO DE ENERGIA DE QUEM PRODUZ ENER GIA: ESTUDANDO O ESTRESSE OCUPACIONAL E SEUS EFEITOS EM GESTORES DE UMA EMPRESA BRASILEIRA DO SETOR DE ENERGIA ELÉTRICA
}

ENERGY CONSUMPTION OF THOSE WHO PRODUCE ENERGY: STUDYING OCCUPATIONAL STRESS AND ITS EFFECTS ON MANAGERS OF A BRAZILIAN COMPANY IN THE ELECTRICITY SECTOR

\section{EL CONSUMO DE ENERGÍA DE QUIEN PRODUCE ENERGÍA: ESTUDIANDO EL ESTRÉS} OCUPACIONAL Y SUS EFECTOS EN GESTORES DE UNA EMPRESA BRASILEÑA DEL SECTOR DE ENERGÍA ELÉCTRICA

Clarissa Daguer Braga

Mestre

clarissadaguer@gmail.com

Luciano Zille

Doutor

Faculdade Novos Horizontes Izille@face.ufmg.br

Antonio Luiz Marques

Doutor

Universidade Federal de Minas Gerais - UFMG marques@face.ufmg.br

Submetido em: 25/03/2012

Aprovado em: 12/03/2014

RESUMO

Tendo em vista o desafio de se manter em um mercado mais exigente, imprevisível e pressionado pelas frequentes mudanças tecnológicas que impactam na produtividade, o profissional de nível gerencial se insere em uma cultura empresarial em que as pessoas trabalham mais horas e mais arduamente, a fim de atingir o sucesso pessoal e as recompensas materiais. O tema "estresse ocupacional e as tensões decorrentes do ambiente ocupacional" ganha importância e proporções ainda maiores do que em décadas anteriores, pois novas variáveis surgiram e as relações entre elas se intensificaram. Com o objetivo de identificar os principais fatores de tensão no trabalho, bem como diagnosticar o nível de estresse ocupacional na função gerencial em uma organização do setor de prestação de serviços em energia elétrica, a presente pesquisa, descritiva e explicativa, utilizando o Modelo Teórico para Explicação do Estresse em Gerentes - MTEG, elaborado por Zille (2005), apontou a sobrecarga de trabalho acarretada pelo uso intensivo de tecnologias organizacionais, incluindo a realização de várias atividades ao mesmo tempo, aliado ao alto grau de cobrança como algumas das principais causas de tensão excessiva no ambiente de trabalho. Também apontou que $62,9 \%$ dos gestores pesquisados apresentam quadro de estresse, variando de estresse leve a moderado a estresse intenso.

PALAVRAS-CHAVE: Estresse Ocupacional. Função Gerencial. Tensão no Trabalho. 


\begin{abstract}
In the context of change, and facing the challenge of staying firm in a demanding and unpredictable market, with frequent technological changes that impact on productivity, management-level professionals are part of a corporate culture in which where people are working harder, and longer hours, to achieve personal success and material rewards. The theme of "occupational stress and the tensions arising from the workplace" gain even greater importance than in previous decades, because new important variables have appeared and the relationships between them have intensified. In order to identify the main factors of stress at work, and to diagnose the level of occupational stress in managers at an electricity company, this descriptive, explanatory study, using the Theoretical Model for Explanation of Stress in Managers MTEG, developed by Zille (2005), points out the work overload caused by the intensive use of organizational technologies, including having to do several activities at the same time, coupled with the high demands, as some of the causes of excessive tension in the workplace. This study also noted that $62.9 \%$ of the managers surveyed had some level of stress, ranging from mild to severe.
\end{abstract}

Keywords: Occupational Stress. Management Function. Tension in the Workplace.

\title{
RESUMEN
}

Considerando el reto de mantenerse en un mercado más exigente, imprevisible y presionado por los frecuentes cambios tecnológicos que impactan sobre la productividad, el profesional de nivel gerencial se inserta en una cultura empresarial en la que las personas trabajan más horas y más arduamente a fin de alcanzar el éxito personal y las recompensas materiales. El tema "estrés ocupacional y las tensiones decurrentes del ambiente ocupacional" cobra importancia y proporciones aún mayores que en décadas anteriores, pues surgieron nuevas variables y las relaciones entre ellas se intensificaron. Con el objetivo de identificar los principales factores de tensión en el trabajo, así como diagnosticar el nivel de estrés ocupacional en la función gerencial en una organización del sector de prestación de servicios en energía eléctrica, la presente investigación, descriptiva y explicativa, utilizando el Modelo Teórico para la Explicación del Estrés en Gerentes - MTEG, elaborado por Zille (2005), apuntó la sobrecarga de trabajo provocada por el uso intensivo de tecnologías organizacionales, incluyendo la realización de varias actividades al mismo tiempo, aliado al alto grado de exigencias, como algunas de las principales causas de tensión excesiva en el ambiente de trabajo. También señaló que el 62,9\% de los gestores investigados presentan cuadro de estrés, variando de estrés leve a moderado hasta estrés severo.

Palabras clave: Estrés Ocupacional. Función Gerencial. Tensión en el Trabajo.

\section{INTRODUÇÃO}

Num ambiente em transformação, as mudanças organizacionais se refletem em mudanças nos diversos níveis da organização e, em especial, nos níveis gerenciais. Entendendo a transformação como um conjunto de mudanças significativas que visam à transformação das estruturas corporativas, os processos de reestruturação provocam mudanças múltiplas, cujos impactos para a gestão podem assumir diferentes formas (ROULEAU, 2005).

Nessa direção, a mudança nas tecnologias de gestão é considerada como aspecto inerente ao cotidiano organizacional e necessária à sobrevivência da empresa no mercado global, competitivo e mutante. O profissional de nível gerencial que se insere nessa nova realidade pertence a uma cultura empresarial em que, segundo Cooper (2005), as pessoas trabalham mais horas e mais arduamente a fim de atingir o sucesso pessoal e as recompensas materiais. Neste momento, uma das fontes de tensão mais evidentes consiste na adoção de novas tecnologias organizacionais, que envolvem 
muito mais do que uma transformação nas normas, estruturas, processos e objetivos da organização. Pressupõe um processo complexo e interdependente, no qual o realinhamento da concepção do homem que trabalha o ambiente físico e o ambiente psicológico que o envolve tornam-se fatores decisivos para aumentar os níveis de tensão no ambiente de trabalho (ZILLE, 2005; ROSSI, 2005; KARASEK; TORRES, 1996; COOPER; SLOAN; WILLIAMS, 1988; COUTO, 1987).

O ritmo frenético da inovação tecnológica, a globalização e a exigência dos mercados consumidores, cada vez mais competitivos, impõem um novo estilo na gestão dos negócios, bem diverso daquele que marcou a economia brasileira em anos anteriores. Mudanças organizacionais acontecem, a todo o momento, em grande parte, sob a égide dos gestores, que passam a lidar com tensões constantes e excessivas no ambiente de trabalho, podendo chegar a desenvolver quadros de estresse ocupacional, entendido aqui como o estresse decorrente das relações que o indivíduo estabelece com seu trabalho.

Para contextualizar as transformações que vêm ocorrendo no ambiente organizacional é importante compreender a interface entre as organizações e as tecnologias de gestão e os seus impactos sobre a função gerencial, apresentados neste trabalho. O estresse ocupacional e as suas abordagens conceituais também são discutidos para que se possa compreender e identificar as principais fontes de tensão, que podem ter como consequência o estresse associado ao trabalho do gerente.

Assim, levando em consideração que um dos grandes desafios que as organizações vêm enfrentando nas últimas décadas consiste em adotar estratégias e tecnologias de gestão que sejam capazes de responder ao contexto de mudança e inovação contínuas, este estudo teve como objetivo geral explicitar as transformações na função gerencial decorrentes da adoção de novas tecnologias organizacionais, buscando identificar as principais fontes de tensão excessiva e as manifestações de estresse ocupacional associadas ao trabalho do gerente. Este objetivo foi atingido a partir de um estudo de caso com característica descritivo- explicativa. A coleta de dados foi realizada por meio do questionário aderente ao Modelo Teórico de Explicação do Estresse Ocupacional em Gerentes MTEG (ZILLE, 2005) e os dados foram tratados estatisticamente a partir da utilização dos softwares Excel e SPSS - Statistical Package for the Social Sciences.

A pesquisa permitiu concluir que a sobrecarga de trabalho acarretada pelo uso intensivo de tecnologias organizacionais, incluindo a realização de várias atividades ao mesmo tempo, aliado ao alto grau de cobrança, foram algumas das principais causas de tensão excessiva no ambiente de trabalho e causadoras de estresse. Também apontou que $62,9 \%$ dos gestores pesquisados apresentam quadro de estresse, variando de estresse leve-moderado a estresse intenso.

Este estudo justifica-se do ponto de vista teórico-prático, contribuindo com dados relevantes sobre as pesquisas relacionadas ao estresse em gerentes, categoria profissional que atua num espaço caracterizado, principalmente, pela ambiguidade de demandas e papéis e pela capacidade de mediar conflitos. Estes profissionais vêm apresentando níveis significativos de tensões no trabalho, sendo acometidos por estresse e doenças decorrentes (LEVI, 2005; DAVEL; MELO, 2005; CHANLAT, 2000; GOLDBERG, 1986).

Considerando que os gestores são agentes fundamentais nas organizações, estudos nesta direção podem contribuir de forma importante para uma melhor compreensão dos problemas enfrentados por estes profissionais. Permite intervenções nas organizações, buscando minimizar os agentes estressores e propiciar aos gestores reagirem de forma positiva diante de situações que geram tensões excessivas no ambiente ocupacional, na busca de melhores condições de trabalho, com reflexos positivos na saúde psíquica e orgânica destes trabalhadores e às organizações contarem com um quadro de profissionais mais aptos ao trabalho.

A sequência deste trabalho está assim organizada: na seção 2 , estabelece o contexto organizacional necessário para o desenvolvimento do estudo; na seção 3, discute-se o tema principal, que é o estresse ocupacional; na seção 4, é apresentada e discutida a parte metodológica; na seção 5 , os resultados são apresentados e discutidos; na seção 6, apresentam-se as principais conclusões que puderam ser extraídas do estudo. 


\section{A FUNÇÃO GERENCIAL E AS NOVAS TECNOLOGIAS DE GESTÃO}

Existem várias explicações sobre a origem da figura do gerente na sociedade. Davel e Melo (2005) destacam três das explicações mais recorrentes: uma explicação técnica, uma da elite e uma da política. Segundo os autores, a explicação técnica sugere que os gerentes apareceram e se desenvolveram como um novo grupo de especialistas por causa do crescimento em escala e em complexidade das empresas capitalistas a partir da metade do século XIX. A explicação da elite diz respeito aos diversos mecanismos sociais e econômicos que um grupo de pessoas (os gerentes) arrogava a si para legitimar-se como tal; e a explicação política observa que a razão pela qual a administração começa a ser desempenhada por administradores especializados tem o propósito de controlar o trabalho.

Chanlat (2000, p. 32) divide o que ele chama de movimento histórico, referindo-se à gestão de empresas, em três grandes momentos: o primeiro, que vai do fim do século XIX até a Segunda Guerra Mundial, quando "a gestão de empresas já não quer ser simplesmente uma arte técnica, ela aspira a transformar-se em ciência". É a época, de acordo com Rossi (2001), do management científico, na qual devem ser destacados autores como Frederic Winslow Taylor, Henry Fayol, Gulick, Urwick, Elton Mayo, entre outros, responsáveis pelas pesquisas importantes dessa época.

O segundo momento mencionado pelo autor pode ser delimitado como aquele que vai do fim dos anos 40 até o início dos anos 80 e assiste ao surgimento da sociedade de consumo de massa, à expansão das consultorias em management e ao grande aumento da oferta de empregos em gestão, devido ao crescimento das empresas e à terceirização da economia. É a era do management moderno. Já o terceiro momento, mencionado por Chanlat (2000, p. 33), é caracterizado a partir dos anos 80 e é

\footnotetext{
(...) marcado por modificações importantes tanto no discurso como no modo de gerenciamento das empresas. A busca de eficácia é principalmente dominada por imperativos financeiros em uma economia de mercado que se globaliza e exige organizações sempre mais competitivas, flexíveis e informatizadas. Os operários, os técnicos e os executivos, cada vez em maior número, conhecem a experiência do desemprego, da precariedade e da instabilidade profissional; sintetizando, estamos na era da gestão estratégica.
}

$\mathrm{Na}$ busca por um melhor arranjo organizacional que possibilite maiores e melhores resultados empresariais, os modelos de gestão caracterizados pelo controle, pela centralização e pela hierarquização passam a ser substituídos por modelos mais flexíveis.

Neste cenário, no contexto brasileiro, os gerentes passam a enfrentar, a partir da década de 1990, situações ambíguas devido aos processos de reestruturação pelo qual passaram várias empresas e às exigências do novo sistema capitalista em construção. A competição se dá de forma intensificada, ocorre a desregulamentação dos mercados, intensifica-se o desenvolvimento das tecnologias de informação e de produção e ocorre a aplicação de novas políticas e legislações governamentais. Aliado a essas transformações, no decorrer da última década e, de forma intensa nos dias atuais, a introdução de novas tecnologias nas organizações impulsionou um amplo redesenho nas estruturas, nos processos e nos modelos de gestão de pessoas.

De acordo com Lemos (2000), a introdução das tecnologias nas organizações traz mudanças que não são apenas mudanças tecnológicas, mas também mudanças de crenças e valores organizacionais que refletem, em grande parte, no desempenho do trabalho de profissionais que se ocupam, entre outras, de funções gerenciais. Os gerentes passam a ter que assumir novas responsabilidades e enfrentar desafios diversos, bem como reformular toda uma lógica estabelecida e, assim, reconstruir um novo modo de pensar relativo aos problemas e aos princípios, às hipóteses, aos arcabouços teóricos e tecnológicos disponibilizados.

O profissional em nível de gestão passa a pertencer a uma cultura empresarial em que, segundo Cooper (2005), as pessoas trabalham mais horas e mais arduamente a fim de atingir o sucesso pessoal e as recompensas materiais. Neste momento, o tema do Estresse Ocupacional ganha importância e proporções ainda maiores do que em décadas anteriores. É a partir da década de 1990, com as mudanças organizacionais mais evidentes e com os novos modelos de gestão, que o gerente vê-se às voltas com uma rotina diária cada vez mais desgastante e se dá cada vez mais 
atenção às tensões decorrentes das pressões excessivas vivenciadas no ambiente de trabalho que muitas vezes têm como consequência o estresse ocupacional (ZILLE, 2005, 2010, 2011a, 2001b; ROSSI, 2005; CHANLAT, 2005; PERREWÉ, 2005; SAUTER; MURPHY, 2005).

Assim, o estresse ocupacional, entendido aqui como o estresse decorrente das relações que o indivíduo estabelece com o trabalho, tornou-se uma fonte importante de preocupação, uma vez que este é reconhecido como um dos riscos mais sérios ao bem-estar psicossocial do indivíduo (FRENCH, 1983, BATEMAN; STRASSER, 1983). Esse tema aparece, então, como assunto de preocupação crescente tanto no meio organizacional quanto no meio acadêmico, quando o assunto em questão é o trabalho do profissional que ocupa função gerencial (ZILLE, 2005; ROSSI; PERREWÉ; SAUTER , 2005).

\section{ESTRESSE OCUPACIONAL}

A palavra inglesa stress, que, para os fins deste trabalho, será grafada em português estresse, é derivada do latim stringere, que significa "apertar" ou "constranger". No século XVII, ganhou conotação de adversidade ou aflição. No final do século seguinte, seu uso evoluiu para expressar força, pressão ou esforço. O conceito de estresse não é novo, mas foi apenas no início do século XX que estudiosos das ciências biológicas e sociais iniciaram a investigação de seus efeitos na saúde física e mental das pessoas, como sendo um estado do organismo após o esforço de adaptação que pode produzir deformação na capacidade de resposta do comportamento mental e afetivo, do estado físico e do relacionamento com as pessoas (NASCIMENTO et al., 1998).

Na primeira metade do século XX, Cannon et al. (1932), fisiologistas da Harvard Medical School, identificaram as reações do organismo humano diante de situações inesperadas, como tensão dos músculos, taquicardia, incapacidade de controlar a respiração e transpiração excessiva como reações ao estresse. Esses autores chamaram o conjunto dessas reações de resposta de luta-ou-fuga, ou seja, o corpo prepara-se ao ser confrontado com uma ameaça para ficar e lutar ou para fugir. Dando continuidade aos estudos de Cannon et al. (1932), Selye (1936, 1956), já em meados do século XX, conseguiu identificar as mudanças que ocorrem na fisiologia corporal quando o indivíduo é exposto a estressores - fatores potencialmente causadores de estresse, ou estímulo capaz de ativar a reação de luta-ou-fuga. Esse autor resumiu a reação ao estresse como um processo de três fases - reação de alarme, fase de resistência e fase de exaustão, denominado-a de síndrome de adaptação geral. Selye (1956) definiu o estresse como a resposta inesperada do corpo a qualquer estímulo que seja solicitado, correspondendo a situações às quais o organismo deve se adaptar.

Nas décadas seguintes, outros pesquisadores somaram-se ao trabalho de Cannon et al. (1932) e Selye $(1936,1956)$ para lançar luz às questões relacionadas ao tema que vinha sendo estudado. No final da década de 1970, Albrecht (1979, p. 113) definiu o estresse como "o conjunto de condições bioquímicas do corpo humano, refletindo a tentativa do corpo de fazer o ajuste às exigências do meio". Assim, o estresse, na visão deste autor, não é tratado como uma doença, mas sim como uma condição de descontrole da função fisiológica normal do corpo humano.

Os estudos estão avançando, chamando atenção de pesquisadores em várias disciplinas, da medicina à administração, cada uma delas usando seus próprios modelos de análise, que acabam por gerar diversos estudos sobre o fenômeno (DOLAN, 2006).

Destacam-se os estudos de Lipp (2005), que acredita que, antes da fase de exaustão preconizada por Selye (1956), existe um período de transição, em que a pessoa não mais está sendo capaz de resistir, mas ainda não atingiu a exaustão completa. Esta fase foi denominada pela autora de 'quase exaustão', na qual as defesas estão se quebrando, caracterizando-se por um meio termo entre a fase de resistência e a de exaustão. Ocorre o enfraquecimento do indivíduo que não está mais conseguindo se adaptar ou resistir aos estressores, estando presente a ansiedade de forma importante, com os indivíduos experimentando uma 'gangorra emocional'.

Na visão de Limongi-França e Rodrigues (2005, p. 36), o estresse deve ser entendido como "uma relação particular entre uma pessoa, seu ambiente e as circunstâncias as quais está submetida, que é avaliada pela pessoa como uma ameaça ou algo que exige dela mais que suas próprias habilidades ou recursos e que põe em perigo seu bem-estar ou sobrevivência". Nesta visão do estresse, foi incluído o 'fator subjetivo' como avaliador do ambiente para posterior reação do indivíduo ao contexto 
no qual está inserido. Desta forma o modelo de análise está relacionado ao estúmulo, à avaliação subjetiva e como decorrência, à resposta.

Para Sant'Anna e Kilimnik (2011), o estresse, entendido como disfuncional, é um desgaste do organismo humano provocado por uma tensão crônica, ou uma tensão aguda de grande intensidade. O quadro de estresse indica que o indivíduo poderá estar adoecendo e requer, portanto, intervenções terapêuticas para minimizar e combater a sintomatologia apresentada, assim como enfrentar de maneira mais positiva as situações vivenciadas.

Com as tendências de reestruturação do trabalho e do emprego, é possível observar que, de acordo com Zille (2005, p.61),

[...] as sociedades estão passando por um processo de intensificação do ritmo em que as mudanças acontecem. Aliado a essa conjuntura, verifica-se uma deterioração da qualidade de vida dos indivíduos. Dessa forma, o estresse apresenta-se como uma variável importante, que vem atingindo os indivíduos de forma geral. Cada período da história contribui de maneira positiva para o desenvolvimento global, mas cobra um preço por esse benefício, sendo o estresse um dos preços mais habituais da atual época de turbulência sociocultural por que passa a humanidade.

Nesse cenário, o estresse no trabalho fica cada vez mais em evidência e recebe cada vez mais destaque, podendo ser entendido, de acordo com Brief, Schuler e Sell (1981), como uma condição que surge a partir da interação dos indivíduos com o trabalho, caracterizado pelas mudanças que acontecem com as pessoas quando são forçadas a desviar de suas funções de rotina.

No caso específico dos gerentes, por natureza do papel, estão expostos a significativos níveis de pressão no ambiente ocupacional que podem gerar tensões excessivas e, por sua vez, ser responsáveis por quadros de estresse ocupacional. Neste caso, entre as fontes de pressão no trabalho estão fatores intrínsecos e extrínsecos a esse, tais como: condições de salubridade; ritmo, incluindo ai as horas excedentes de trabalho; natureza e conteúdo do trabalho; papel do indivíduo na organização; relacionamento interpessoal; carreira e realização; estrutura e clima organizacional; e interface casa/trabalho (COOPER, 2005; COOPER et al. 1988).

\section{As abordagens conceituais do estresse ocupacional}

As abordagens bioquímica, psicológica e sociológica podem ser consideradas complementares e interligadas, sendo consideradas as principais abordagens conceituais referentes ao estresse ocupacional. A estrutura a seguir apresentada está de acordo com a organização desenvolvida por Veloso (2000).

A abordagem bioquímica, ou seja, a fisiologia do estresse surgiu nas décadas de 1930 a 1950, influenciada pelos estudos de Selye (1936), primeiro pesquisador a estudar o estresse do ponto de vista bioquímico. $O$ trabalho de Selye foi influenciado por pesquisadores que não estudaram diretamente o estresse, mas que proporcionaram importantes subsídios para a sua compreensão, como foi o caso do fisiologista francês Bernard, ao apontar a capacidade de manter a constância do equilíbrio interno como um dos traços mais característicos de todos os seres vivos e do fisiologista Cannon et al. (1932), que retomou o conceito de Bernard sobre a constância do equilíbrio interno, desenvolvendo-o e denominando-o de homeostase. Avançando em relação às constatações desses dois fisiologistas, Selye (1936), além da reação de alarme, descreveu outras duas fases, a de resistência e a de exaustão. Para este autor, decorrentes do estado de tensão, diversas reações fisiológicas ocorrem no organismo para prepará-lo para enfrentar e/ou adaptar-se às fontes de pressão excessiva ao qual o organismo está sendo submetido.

A abordagem psicológica, que apresenta sua ênfase principal na compreensão da influência que a percepção e o comportamento do indivíduo são manifestados no processo de estresse, apresenta as seguintes vertentes: psicossomática; interacionista; behaviorista; psicopatologia do trabalho e a da psicologia social. Essas vertentes mostram que a abordagem psicológica, em relação aos estudos sobre estresse, não apresenta uma direção única, embora nas suas diversas vertentes apresente coerência em relação a determinados pressupostos. Importantes contribuições para o desenvolvimento desta abordagem do estresse foram realizadas com base nas pesquisas de Cooper, Cooper e Eaker (1988), uma vez que estes estudos ampliaram o entendimento sobre o complexo 
processo que envolve os seres humanos. No entanto, na visão de Cooper, Cooper e Eaker (1988), Lhuilhier, Mignèe e Raix (1990), Arnald, Robertson e Cooper (1991) apud Veloso (2000), o trabalho de Lazarus (1974) é considerado um marco importante para os estudos da abordagem psicológica, uma vez que o mecanismo psicológico é percebido como determinante no processo, associando-se ao desencadeamento de quadros de estresse.

Já a abordagem sociológica está relacionada à compreensão das diversas variáveis que se estabelecem no contexto da sociedade. Nesta abordagem, de acordo com Cassirer (1994), percebese uma dependência entre a visão de mundo do indivíduo e a sua realidade social. Conforme afirma Leontiev (1978), apud Veloso (2000), o desenvolvimento psíquico dos indivíduos está relacionado à estrutura cultural estabelecida e, nessa dimensão, as alterações culturais afetam diretamente os mecanismos psicológicos individuais. Nos dias atuais, a presença mais forte do estresse social revela uma alteração sociocultural que influencia o mecanismo psíquico e altera as condições ambientais nas quais o indivíduo está inserido, provocando influência no mecanismo biológico. Sendo assim, para a compreensão do estresse, é de fundamental importância entender não só o indivíduo, no que se refere a seu mecanismo psicológico e a seu ambiente, como também os valores sociais e as suas transformações.

Essas abordagens permitem uma visão global das manifestações do estresse, sendo que a abordagem bioquímica permite uma observação principalmente no que se refere ao aspecto fisiológico, a abordagem psicológica permite entender a importância dos mecanismos psicológicos no desencadeamento de quadros de estresse e a abordagem sociológica mostra a importância de se compreender as novas variáveis que se estabelecem dentro da sociedade para que se possa, então, interpretar as fontes de pressão à luz de cada nova realidade. Essas abordagens conceituais mostram sob diversas óticas como podem ser entendidas e estudadas as manifestações relacionadas ao estresse, constituindo-se em importante base teórica para realização de estudos nessa área, como é o caso do presente trabalho, que foca de forma principal na abordagem psicológica.

Mais recentemente, Zille (2005) desenvolveu um Modelo Teórico de Explicação do Estresse Ocupacional em Gerentes (MTEG), tendo como referência Couto (1987); Cooper et al. (1988); Karasek (1998); Chanlat (1996). De acordo com o autor, a manifestação do estresse está relacionada ao desequilíbrio acentuado entre os níveis de tensão que o indivíduo recebe do meio e à sua capacidade psíquica de suportá-lo.

A seguir será apresentado, com base na Figura 1, o Modelo Teórico utilizado neste trabalho para explicar o estresse ocupacional em gerentes (MTEG).

O modelo em referência é composto por cinco construtos de primeira ordem (fontes de tensão no trabalho; fontes de tensão do indivíduo e do papel gerencial; mecanismos de regulação; sintomas de estresse; e indicadores de impacto na produtividade). Cada construto de primeira ordem é explicado pelos construtos de segunda ordem, que por sua vez estão relacionados aos seus respectivos indicadores, que compõem o questionário aderente ao Modelo Teórico de Explicação do Estresse Ocupacional em Gerentes (MTEG), utilizado nesta pesquisa. A exceção se faz ao construto indicadores de impacto na produtividade, que diretamente se relaciona aos indicadores.

Como podem ser observadas na Figura 1, as fontes de tensão no trabalho são explicadas por três construtos que são processos de trabalho; relações no trabalho; insegurança na relação de trabalho e convivência com indivíduos de personalidade difícil. O construto fontes de tensão do indivíduo e do papel gerencial, por sua vez, é explicado por responsabilidades acima dos limites; estilo e qualidade de vida; aspectos específicos do trabalho do gerente; e desmotivação. Já o construto mecanismos de regulação é explicado por interação e prazos; descanso regular e experiência no trabalho; e atividade física. O construto sintomas de estresse é explicado pelos construtos de segunda ordem sintomas de hiperexcitabilidade e senso de humor; sintomas psíquicos, do sistema nervoso simpático e gástrico; e sintomas de aumento do tônus, tontura/vertigem, falta ou excesso de apetite e relaxamento. $O$ construto indicadores de impactos na produtividade é explicado de forma direta pelos seus respectivos indicadores, que são em número sete (ZILLE, 2005, p. 191). 


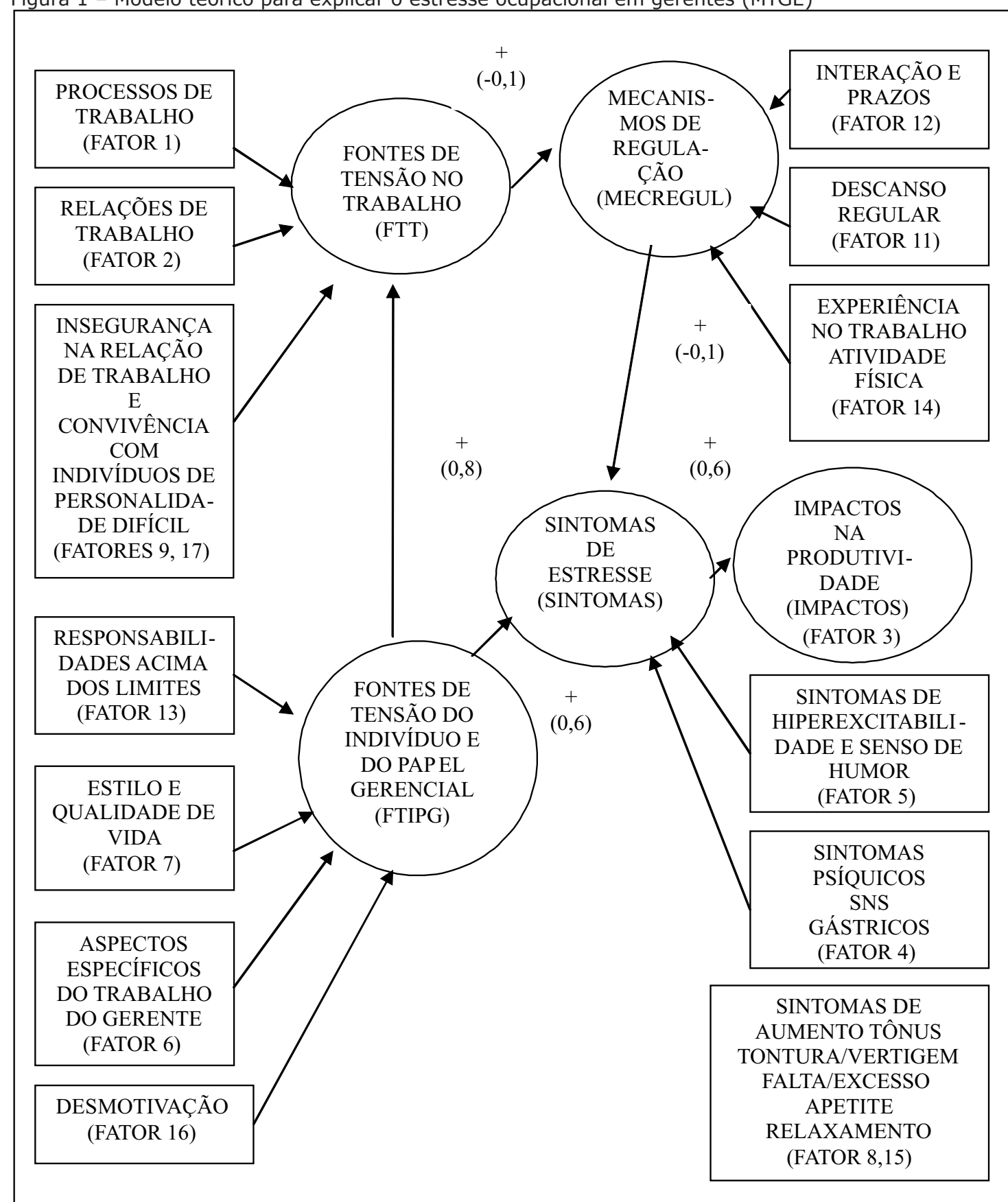

Fonte: Zille (2005, p.191).

Legenda: 1) As indicações dos valores entre parênteses no modelo teórico como $+(0,8) ;+(-0,1) \ldots$. referem-se aos coeficientes de caminho estimados pelo PLS - Partial Least Squares, como intervalos de confiança a 5\%, para explicação dos construtos do modelo teórico. 2) A numeração relativa aos fatores dos constructos de segunda ordem constantes do modelo teórico, referem-se aos indicadores dos respectivos fatores extraídos por meio da Matriz Rotacionada de Componentes Final (ZILLE, 2005 , p. 186 e 148-149).

\section{METODOLOGIA E CARACTERIZAÇÃO DA ORGANIZAÇÃO PESQUISADA}

Quanto ao tipo e ao método de pesquisa, quanto aos fins, caracteriza-se por ser uma pesquisa explicativa, cujo objetivo é identificar os fatores que contribuem, de alguma forma, para a ocorrência do fenômeno de estresse ocupacional em um grupo de gestores; e descritiva, na medida em que apresenta as características de determinada população/fenômeno ou, mesmo, estabelece relação 
entre as variáveis estudadas. Quanto aos meios de investigação, trata-se de pesquisa de campo e estudo de caso.

A pesquisa de campo está diretamente relacionada a uma investigação empírica realizada na organização onde ocorreu o fenômeno objeto de investigação. O estudo de caso buscou aprofundar a análise e a descrição considerando as transformações na função gerencial propostas pela literatura, as mudanças nas tecnologias organizacionais nas últimas décadas e as principais fontes de tensão no ambiente de trabalho que acometem os gestores da empresa em estudo, responsáveis pelo desencadeamento de quadros de estresse no grupo ocupacional pesquisado (VERGARA, 2010; GONÇALVES; MEIRELLES, 2004).

O presente trabalho teve como unidade de análise as práticas gerenciais e como unidade de observação os indivíduos que constituem os níveis gerenciais: gerentes e superintendentes de uma organização com atuação na área de prestação de serviços no setor de energia elétrica da região sudeste brasileira.

Foram considerados dois grupos - gerentes e superintendentes - em função de sua representação na gestão da empresa pesquisada, da proximidade da função que exercem e do fato de vivenciarem todo o processo de mudança organizacional ao qual a empresa foi submetida nos últimos anos.

Para coleta de dados, utilizou-se o questionário referente ao MTEG (ZILLE, 2005). O instrumento buscou detectar as principais fontes de tensão presentes no ambiente de trabalho e o nível de estresse ao qual estão submetidos os gerentes da organização pesquisada, medindo a exposição potencial em relação aos estressores presentes nas diversas áreas de trabalho.

Para a aplicação do questionário, utilizou-se o software Limesurvey. O questionário ficou disponível exclusivamente para os gestores que receberam o convite para preenchê-lo via web no link específico para o Limesurvey (http://daguer.psc.br/limesurvey/index.php?sid =72499\&lang=pt-BR).

Após a aplicação do questionário, os dados foram tratados e processados utilizando-se os softwares Excel e SPSS - Statistical Package for the Social Sciences. Foram realizadas as análises descritivas, o teste de frequência, a análise exploratória dos dados e a estatística bivariada - médias e testes Qui-Quadrado.

O teste de inferência estatística Qui-Quadrado, teste de hipóteses que se destina a encontrar um valor da dispersão para duas variáveis nominais, foi utilizado para avaliar a associação existente entre a variável nível de estresse e as variáveis idade, consumo de bebida alcoólica, nível hierárquico, horas trabalhadas e aspectos relacionados à saúde, como apresentados na conclusão deste estudo.

\section{Caracterização da organização pesquisada}

A organização pesquisada é, hoje, uma das maiores e mais importantes concessionárias de energia elétrica do Brasil, por sua posição estratégica, competência técnica e mercado atendido. Sua área de concessão cobre, principalmente, a região Sudeste do Brasil. Possui 54 usinas em operação, 5 delas em sistema de parcerias com grupos empresariais, com base predominantemente hidrelétrica, que produzem energia para atender a mais de 17 milhões de pessoas em 774 municípios. Além dessas, outras 3 usinas estão em fase final de construção.

Caracteriza-se como uma organização de economia mista, sendo que o setor privado externo e o setor privado interno possuem, respectivamente, 4,2\% e 11,53\% do controle acionário; possui atualmente 10.863 empregados, que atuam, principalmente, em três grandes áreas: geração, distribuição e transmissão de energia. Do número total de empregados, 9.389 , ou $86,5 \%$, são homens e 1.474 , ou $13,5 \%$, são mulheres.

Considerou-se esta organização para o desenvolvimento deste estudo, por pertencer a um setor que atua com alta tecnologia e que vem passando por importantes transformações em sua estrutura organizacional, o que reflete diretamente no trabalho dos empregados, especialmente dos gestores, que estão permanentemente se adaptando às novas situações para sobreviverem na organização. Vem constantemente inovando e investindo em tecnologias de gestão, tendo adotado nos últimos anos o sistema integrado de gestão SAP R/3, projetos de automação de grandes instalações, novas redes de transmissão de dados, projeto Gemini de ferramentas de geoprocessamento para 
aperfeiçoamento do sistema elétrico, como também o sistema Omnisat para comunicação com veículos mais próximos do atendimento ao usuário.

\section{ANÁLISE E APRESENTAÇÃO DOS RESULTADOS}

Após o tratamento estatístico, os dados foram analisados levando-se em consideração as transformações na função gerencial e as mudanças nas tecnologias organizacionais que ocorreram nas últimas décadas com importante impacto no setor de energia, que é o foco do presente estudo.

O estudo se deu com 62 , ou $38,75 \%$, dos 160 gerentes e superintendentes da empresa, sendo que, do total da amostra, 58 , ou $93,56 \%$, são gerentes e 4 , ou $6,44 \%$, são superintendentes.

Com relação à idade, $72 \%$ da amostra distribuem entre as idades de 40 a 50 anos; e quanto ao tempo de atuação na função gerencial, na empresa pesquisada, localiza-se, principalmente, em dois extremos: 21 , ou 33,9\%, exercem a função gerencial há até 2 anos e outros 21 , ou 33,9\%, há mais de 9 anos; 16, ou 25,8\%, de 3 a 5 anos; e 4, ou 6,4\%, de 6 a 9 anos.

Em relação ao número de horas trabalhadas, considerando que os profissionais são contratados para trabalhar 40 horas por semana, 41,9\% afirmaram que trabalham entre mais de 40 e 50 horas; e entre mais de 50 e 60 horas semanais, 48,4\%. Esses dados denunciam que estes profissionais estão trabalhando, em média, de 10 a 12 horas por dia, o que pode caracterizar uma sobrecarga de trabalho, induzindo o estresse, conforme afirmam Cooper (2005) e Cooper et al. (1988).

Quando o assunto é consumo de bebidas alcoólicas, do total da amostra 53, ou 85,5\%, afirmam que as consomem. Quanto aos cuidados com a saúde, 56, ou 90,4\%, dos 62 gestores pesquisados fazem check-up anualmente. $52 \%$ da amostra apresentam algum tipo de problema de saúde, como hipertensão, diabetes, gastrite, colesterol alto, hipotireoidismo, obesidade, esofagite, dislipidemia, problema na coluna vertebral e refluxo gástrico. Verifica-se, portanto, que a quase totalidade das ocorrências identificadas em relação à saúde é relacionada pela literatura, direta ou indiretamente, com quadros de estresse (ALBRECHT, 1979; COOPER, C. 1988; COUTO, 1987, 2007; KARASEK, 1986, 1988; LEVI, 2005). Estes dados confirmam achados anteriores e reforçam a importância de ações preventivas em relação ao estresse, que pode gerar adoecimento dos trabalhadores, tendo consequências importantes não só para os indivíduos, como também para o contexto ocupacional, sobretudo, com reflexos na produtividade.

\section{Diagnóstico do estresse}

Para análise do nível de intensidade de estresse ocupacional apontado pela pesquisa, tomouse como referência a escala desenvolvida e validada por Zille (2005, p. 222, 223): Ausência de Estresse < 1,75; Estresse Leve a Moderado $\geq 1,75$ a $<2,46$; Estresse Intenso $\geq 2,46$ a $<3,16$; e Estresse Muito Intenso $\geq 3,16$.

Os conceitos utilizados em relação à categorização do estresse foram os seguintes: a) Ausência de Estresse - significa um estado de bom equilíbrio entre as demandas psíquicas advindas do ambiente, tais como trabalho, família e social, entre outras, e a estrutura psíquica do indivíduo. b) Estresse Leve a Moderado - indica a ocorrência de manifestações de estresse, no entanto em grau compensado, podendo não gerar impactos importantes nos diversos ambientes de interação do indivíduo. c) Estresse Intenso - indica a ocorrência de manifestações de estresse num grau elevado, podendo gerar impactos importantes no indivíduo. As condições orgânicas e psíquicas já podem estar apresentando alterações e, em alguns casos, os indivíduos necessitam de tratamento e acompanhamento psicológico, podendo necessitar também de tratamento clínico. d) Estresse Muito Intenso - indica a ocorrência de manifestações de estresse num grau muito elevado, gerando impactos significativos nos diversos ambientes em que o indivíduo opera. As condições orgânicas e psíquicas apresentam alterações importantes e os casos dessa intensidade necessitam de tratamento e acompanhamento clínico e/ou psicológico (ZILLE, 2005).

Os níveis de estresse identificados na amostra global, de acordo com a caracterização definida, são apresentados a seguir por meio da Tabela 1: 
Tabela 1: Diagnóstico de estresse para a amostra global

\begin{tabular}{llll}
\hline \multicolumn{2}{l}{ Diagnóstico de Estresse para a Amostra Global } & & \\
\hline Níveis de Estresse & Valor de Referência & Quantidade & $\%$ \\
\hline Ausência de Estresse & $<1,75$ & 23 & 37,1 \\
Estresse Leve a Moderado & $>$ ou $=1,75$ a $<2,46$ & 32 & $\mathbf{5 1 , 6 1}$ \\
Estresse Intenso & $>$ ou $=2,46$ a $<3,16$ & 7 & $\mathbf{1 1 , 2 9}$ \\
Estresse Muito Intenso & $>$ ou $=3,16$ & 0 & 0 \\
TOTAL & - & 62 & 100,00
\end{tabular}

Fonte: Dados da pesquisa (2007).

De acordo com os dados constantes na Tabela 1,62,9\%, ou 39 gestores, apresentam quadro de estresse, variando de leve/moderado a estresse intenso. Estes dados apontam que ocorre desequilíbrio entre a estrutura psíquica e as pressões psíquicas advindas das situações de trabalho vivenciadas por estes gestores. Por outro lado, 37,1\%, ou 23, apresentam ausência de estresse.

Esse resultado vai ao encontro das constatações das últimas pesquisas realizadas no Brasil sobre 0 tema. Em pesquisa realizada por Zille (2005) em 15 organizações brasileiras representativas de vários segmentos da economia nacional, com 550 ocupantes da função gerencial nos seus diversos níveis hierárquicos, $63 \%$, ou 345 , dos indivíduos foram diagnosticados como estressados, sendo que $18 \%$, ou 98 , apresentaram quadro de estresse bastante agravado, não reunindo, portanto, as condições de saúde psíquicas e orgânicas necessárias à execução das suas atividades normais de trabalho.

Confirmando esses resultados, em pesquisa realizada pelo ISMA (2003), verifica-se a incidência de estresse muito intenso em relação aos trabalhadores brasileiros. Em situação mais crítica estão os profissionais japoneses e com índices menores do que os identificados no Brasil (70\%) estão China, Estados Unidos, Alemanha, França e Israel. A pesquisa também revela que os altos índices de estresse estão relacionados com as fusões e com o 'enxugamento' do quadro de pessoal das empresas nos últimos anos. Outra pesquisa realizada pela ISMA-Brasil, em 2004, apresenta dados semelhantes com trabalhadores brasileiros que atuavam em diversas ocupações, a qual revelou que $70 \%$ dos pesquisados apresentavam quadro de estresse, o que também corrobora com a pesquisa de Couto (1987), com amostra de 552 altos executivos, a qual revelou já naquela época que $68 \%$ estavam estressados.

Dados recentes no contexto público brasileiro, relativo ao estado de Minas Gerais, apontam que de um universo de 181 gestores pesquisados, 74\% foram diagnosticados com estresse. Destes, $56 \%$ com quadro de estresse intenso ou muito intenso, o que indica a ocorrência de manifestações de estresse com impactos significativos para os indivíduos no que se refere às condições orgânicas e psíquicas, com implicações diretas no ambiente de trabalho (MAFFIA, 2013).

Quando a análise é realizada por nível hierárquico, os dados podem ser observados, como na Tabela 2:

Tabela 2: Análise do nível de estresse, segundo o nível hierárquico

\begin{tabular}{l|llll|l|l}
\hline \multirow{2}{*}{ Níveis de Estresse } & Nível Hierárquico & & & & $\begin{array}{l}\text { Total por } \\
\text { níveis de } \\
\text { estresse }\end{array}$ & \% \\
\cline { 2 - 7 } & Superintendente & $\%$ & Gerente & $\%$ & & \\
\hline Ausência & 3 & 75,00 & 20 & 34,48 & 23 & 37,10 \\
Leve a moderado & 0 & 0 & 32 & $\mathbf{5 5 , 1 7}$ & 32 & 51,61 \\
Intenso & 1 & $\mathbf{2 5 , 0 0}$ & 6 & $\mathbf{1 0 , 3 5}$ & 7 & 11,29 \\
Muito intenso & 0 & 0 & 0 & 0 & 0 & 0 \\
TOTAL & 4 & 100,00 & 58 & 100,00 & 62 & 100,00 \\
\hline Fonte: Dados da pesquisa (2007). & & & & &
\end{tabular}


Como apresentado na Tabela 2, é possível observar que na amostra pesquisada, predominantemente composta por gerentes (93,56\%), estes apresentam níveis mais elevados de estresse do que os superintendentes, que são gestores que ocupam funções hierarquicamente mais elevadas. Observa-se que $65,62 \%$ dos gerentes apresentam quadro de estresse, em contrapartida a $25 \%$ dos superintendentes.

A maior incidência de quadros de estresse em gerentes, indivíduos que pela natureza da função e por ocuparem cargos intermediários estão mais vulneráveis a um maior nível de pressão no ambiente ocupacional, coincide com os resultados obtidos por Zille (2005). De acordo com a pesquisa realizada pelo autor, o nível de gerência intermediária sofre maior intensidade de pressão advinda do contexto de trabalho, uma vez que, por ocupar posição intermediária, é pressionado não só por seus superiores como também por seus pares e subordinados. Assim, essa é uma posição que, consequentemente, tende a apresentar maior incidência de estresse. A incidência de maior nível de estresse em gestores intermediários também foi constatada por Maffia (2013) em pesquisa realizada com gestores públicos.

Conhecendo o diagnóstico de estresse, a seguir, serão apresentados os dados de acordo com as variáveis inerentes ao modelo teórico (MTEG) utilizado para analisar dos resultados desta pesquisa.

\section{O estresse ocupacional e as demais variáveis do estudo}

Como principal fonte de tensão excessiva presente no trabalho dos gestores com base na análise da amostra global, identificou-se a sobrecarga de trabalho, principalmente acarretada pelo uso das tecnologias como e-mail's, softwares, sistemas integrados de gestão. Este indicador foi apontado por $51,61 \%$ da amostra como sendo frequentemente ou muito frequentemente a principal fonte de tensão excessiva no trabalho dos gestores. O resultado confirma os dados apresentados pela literatura sobre o impacto direto que as novas tecnologias organizacionais exercem no trabalho dos ocupantes da função gerencial. A possibilidade de ser um gerente eficaz está ligada à capacidade de dar o pulo tecnológico e inserir-se ativamente nas novas linhas de trabalho (KLISBERG, 1993; COUTO, 1987; MELO, 2000; MAFFIA, 2013).

Outra fonte de tensão excessiva, destacada por 48,39\% da amostra, foi a realização de várias atividades ao mesmo tempo, com alto grau de cobrança. Essa também é uma característica do trabalho dos gestores, como destacado por Melo (2000), ao lembrar que o gerente passa a ser caracterizado por um estilo de atuação aberto, integrador de diversos ângulos de análise, com múltiplos cenários simultâneos, o que, na maior parte das vezes, gera neste profissional grande sobrecarga de trabalho.

Muitos prazos e prazos apertados, número excessivo de horas de trabalho e excessiva carga de trabalho, muitas vezes ultrapassando os limites, constituem outras fontes importantes de tensão identificadas neste segmento ocupacional. Estes dados são apontados por Cooper (2005) e Cooper et al. (1988).

Aprofundando a análise em relação aos gestores que apresentaram 'estresse intenso', tem-se que a principal fonte de tensão excessiva no trabalho para esses profissionais é a realização de várias atividades ao mesmo tempo, com alto grau de cobrança. Neste grupo, a tecnologia também é destacada como fonte de tensão excessiva, acompanhada da rotina de trabalho, com muitos prazos e prazos apertados, como também o número excessivo de horas trabalhadas, chegando a uma média diária de 10 a doze horas.

Com relação aos Outros aspectos específicos relacionados ao trabalho, também causadores de tensão excessiva, foram identificados os seguintes: assumir compromissos muito desafiadores no contexto do trabalho e levar a vida de forma muito corrida, realizando cada vez mais trabalho em menos tempo, mesmo quando não há exigências para tal. Estas situações foram apontadas por $54,84 \%$ dos gestores.

Os principais sintomas de estresse apresentados como presentes frequentemente e muito frequentemente foram a fadiga, dor nos músculos do pescoço e dos ombros, nervosismo acentuado, 
insônia, ansiedade, irritabilidade fácil e falta ou excesso de apetite. Esses dados confirmam pesquisas realizadas por Couto (1987); Zille (2005, 2010, 2011a, 2011b,); Maffia (2013).

Com relação aos mecanismos de regulação, ou seja, ações utilizadas pelos gestores para minimizar ou neutralizar situações tensionantes, destaca-se a possibilidade de atrasar os cronogramas de trabalho, apontado por $69,35 \%$ dos que afirmaram que algumas vezes é possível. Para $51,61 \%$ dos pesquisados é possível, quando necessário, questionar os cronogramas de trabalho. 45,16\% afirmaram que na maioria das vezes é possível gozar férias regulares e que é sempre possível ter descanso semanal regular. É importante destacar que a ausência de quadros de estresse muito intenso na amostra pesquisada pode ter sido influenciada, em grande parte, pelos mecanismos de regulação usados pelos gestores para lidarem com as fontes de tensão excessiva no trabalho. De acordo com Couto, Herkenhoff e Lima (2007), muitas vezes, o determinante da existência ou inexistência de estresse diante das mesmas situações vivenciadas por gestores diversos está na existência ou não de mecanismos de regulação que possibilitam a retomada do equilíbrio e da integridade física, cognitiva e mental.

Quando o construto indicador de impactos na produtividade é analisado, tem-se que $45,16 \%$ da amostra afirmam que, algumas vezes, frequentemente ou muito frequentemente, nos últimos três meses, têm passado por um processo de diminuição da produtividade. Os principais indicadores destacados foram a dificuldade de lembrar fatos recentes relacionados ao trabalho que anteriormente eram facilmente lembrados; dificuldade de concentração no trabalho; e perda do controle sobre os eventos da vida, como trabalho, família e relacionamentos diversos. Estes dados confirmam as pesquisas realizadas por Zille e Braga (2010) e por Zille, Braga e Zille (2011), em que os grupos de gerentes diagnosticados com estresse apresentaram, com maior frequência, os indicadores: dificuldades em lembrar fatos recentes relacionados ao trabalho que anteriormente eram lembrados com naturalidade; perda de controle em relação aos eventos da vida; e redução da eficiência no trabalho.

A seguir serão apresentadas as conclusões que puderam ser inferidas da pesquisa e algumas recomendações.

\section{CONCLUSÕES}

O objetivo central deste trabalho, de explicitar as transformações na função gerencial decorrentes da adoção de novas tecnologias organizacionais, buscando identificar as principais fontes de tensão e as possíveis manifestações de estresse ocupacional associadas ao trabalho do gestor, foi atingido a partir de um estudo de caso com característica descritivo-explicativa, com uma amostra de 62 gerentes/ superintendentes de uma organização do setor de prestação de serviços em energia elétrica.

Os resultados apontam que, dos 62 gestores pesquisados, 39, ou 62,9\%, apresentam quadro de estresse ocupacional, sendo que destes, 32 , ou $51,61 \%$, apresentam quadro de estresse leve a moderado e 7, ou 11,29\%, apresentam quadro de estresse intenso. Apenas 37,1\% da amostra, o que corresponde a 23 indivíduos, apresentaram ausência de estresse.

Com relação aos sintomas, os dados coincidem com os destacados por Zille (2005) e Couto (1978) para os casos de indivíduos que apresentam quadros de estresse. Assim como nas pesquisas realizadas pelos autores, na presente pesquisa os sintomas identificados com maior frequência foram a fadiga, dor nos músculos do pescoço e dos ombros, nervosismo acentuado, insônia, ansiedade e falta ou excesso de apetite.

Com relação às principais fontes de tensão que estão afetando os gestores, pode-se constatar que, na amostra global, a principal fonte de tensão excessiva foi a sobrecarga de trabalho acarretada pelo uso frequente das tecnologias de gestão (e-mail, softwares, sistemas integrados de gestão, entre outros). Outra fonte de tensão excessiva identificada foi a execução de várias atividades ao mesmo tempo, com alto grau de cobrança, como também a definição de muitos prazos e prazos apertados como rotina no trabalho. Identificou-se também a execução de trabalho complexo, que muitas vezes deixa o gestor desgastado e, finalmente, um número excessivo de horas trabalhadas.

Para o grupo diagnosticado com estresse intenso, a principal fonte de tensão excessiva foi a realização de várias atividades ao mesmo tempo, com alto grau de cobrança. Neste grupo, a tecnologia 
também é destacada, acompanhada de rotina de trabalho com muitos prazos e prazos apertados, acarretando grande incômodo e tensão, além do número excessivo de horas trabalhadas.

Ao se realizar análise comparativa das fontes e nível de estresse em relação às variáveis tempo na função, nível hierárquico, jornada de trabalho e aspectos de saúde, constatou-se que, quanto ao tempo em que o profissional atua na função de gestor, para a amostra global, os dados permitem traçar uma perspectiva de que há possibilidade de que o nível de estresse se mantenha durante a carreira gerencial, independente do tempo que o profissional ocupe o cargo em questão. Em relação ao grupo de gestores que apresenta estresse intenso, os dados apontam para a mesma situação do que foi observado em relação à amostra global.

Com relação ao nível hierárquico, foi possível observar que, dos gerentes, a maior parte apresenta quadro de estresse, ao passo que a maior parte dos superintendentes apresenta ausência de estresse. Dos quadros de estresse intenso, $85,7 \%$ foram diagnosticados em gerentes e $14,3 \%$ foram diagnosticados em superintendentes. Esses dados confirmam a constatação de Zille (2005) de que os ocupantes de níveis intermediários de gerência estão mais propensos a desenvolver quadros de estresse, uma vez que, por ocuparem posição intermediária, são acometidos com maior frequência pelas pressões que vêm não só de seus superiores como também de seus subordinados e pares.

Os dados permitem concluir também que, para esta pesquisa, o estresse ocupacional não está diretamente relacionado com a progressão hierárquica da função, uma vez que os superintendentes, que ocupam funções hierarquicamente superiores, encontram-se menos estressados que os gerentes. Aqui, pode existir uma perspectiva otimista de redução do nível de estresse ao alcançar uma posição superior na escala hierárquica, se esta for uma progressão natural dentro da empresa.

Sobre a jornada de trabalho, foi possível constatar que a maior parte da amostra trabalha além da carga horária para a qual é contratada. Este resultado confirma a posição de Cooper (2005) para os ocupantes da função gerencial da atualidade. Segundo o autor, o profissional de nível gerencial que se insere na nova realidade pertence a uma cultura empresarial em que as pessoas trabalham mais horas e mais arduamente, a fim de atingir o sucesso pessoal e as recompensas materiais.

Com relação à saúde, foi possível constatar que os gestores que apresentam problemas de saúde são mais propensos a desenvolver quadros de estresse do que aqueles que não o apresentam, ressaltando que há possibilidade de os problemas serem decorrentes do estresse ocupacional vivenciado. Esses dados são confirmados pelo teste de inferência estatística, que confirma que há relação de dependência direta entre problemas de saúde e nível de estresse que os gestores apresentam $(p>0,05)$.

Pôde-se identificar que os profissionais que estão mais propensos a desenvolver quadro de estresses intenso são aqueles que hierarquicamente ocupam o cargo de gerente, trabalham de acima 40 a 60 horas semanais e possuem algum problema de saúde, mesmo que este não tenha se desenvolvido em função do estresse.

Os resultados apresentados requerem atenção por parte não só dos indivíduos como também da organização. Para esta, é importante salientar que a manutenção de quadros de estresse contínuo relacionado ao trabalho pode gerar alguns prejuízos, como queda na produtividade ou, até mesmo, afastamento de alguns profissionais por problemas relacionados à saúde física e psíquica.

De acordo com Levi (2005), o estresse contínuo relacionado ao trabalho constitui um importante fator determinante dos transtornos depressivos, além de ser um fator determinante do aumento da pressão sanguínea, provavelmente contribuindo para a morbidade de doenças cardíacas e do desencadeamento de quadros de diabetes. Outras consequências podem ser: queda de produtividade dos gerentes, exaustão, perda de peso, esgotamento físico e aparecimento de outras doenças, incluindo os distúrbios cardiovasculares como moléstias do coração, AVC's e problemas renais devido à hipertensão arterial.

Este foi um estudo que buscou contribuir com as pesquisas sobre estresse em gestores, uma vez que os níveis de estresse que vêm sendo apresentados por esta categoria profissional mostramse cada vez mais evidentes e, de certo ponto, alarmantes. Assim, alternativas para diminuir essas ocorrências e melhorar as condições de trabalho dos gestores são focos importantes para pesquisas futuras sobre o tema. Outra direção importante para pesquisas subsequentes é a investigação das diferenças de níveis e manifestações de estresses ressaltando o gênero. 
ALBRECHT, K. O gerente e o estresse: faça o estresse trabalhar para você. Rio de Janeiro: Zahar, 1979.

ARNOLD, J.; ROBERTSON, I. T.; COOPER, C. L. Work psychology: understanding human behavior in the workplace. London: Pitman, 1991.

BATEMAN, T. S.; STRASSER, S. A cross-lagged regression test of the relationship between job tension and employee satisfaction. Journal of Applied Psychology, v. 68, p. 439-445, 1983.

BRIEF, A. P.; SCHULER, R. S.; VAN SELL, M. Managing job stress. Boston: Little, Brown, 1981.

CANNON, W. B. et al. The wisdom of the body. New York: W.W. Norton, 1932.

CASSIRER, E. Ensaio sobre o homem: introdução a uma filosofia da cultura humana. Tomás Rosa Bueno (trad.) São Paulo: Martins Fontes, 1994.

CHANLAT, J. F. Modos de Gestão, Saúde e Segurança no Trabalho. In: DAVEL, E.; VASCONCELO, J. (Orgs.). Recursos humanos e subjetividade. São Paulo: Vozes, 1996.

CHANLAT, J. F. Ciências sociais e management: reconciliando o econômico e o social. São Paulo: Atlas, 2000.

CHANLAT, J. F. Mitos e realidade sobre estresse dos gerentes. In: DAVEL, E. P. B; MELO, M. C. de O. L. Gerência em ação: singularidades e dilemas do trabalho gerencial. Rio de Janeiro: FGV, 2005.

COOPER, C. et al. Occupational stress indicator: test sources of pressure in job. Austin: Windsor, 1988.

COOPER, C. L.; COOPER R.D.; EAKER, L. Living with stress. London: Penguin Books, 1988.

COOPER, C. L.; SLOAN, S. J.; WILLIAMS, S. Occupational Stress Indicator: Management Guide. Windsor, UK: NFER-Nelson, 1988.

COOPER, C. L. A natureza mutante do trabalho: o novo contrato psicológico e os estressores associados. In: ROSSI, A. M.; PERREWÉ, P. L.; SAUTER, S. L. (Org.). Stress e qualidade de vida no trabalho: perspectivas atuais da saúde ocupacional. São Paulo: Atlas, 2005.

COUTO, H. A. Stress e qualidade de vida dos executivos. Rio de Janeiro: COP, 1987.

COUTO, H. A.; HERKENHOFF, F. L.; LIMA, E. G. Estresse ocupacional e hipertensão arterial sistêmica. Revista Brasileira de Hipertensão, v. 14, p. 112-115, 2007.

DAVEL, E.; MELO, M. C. O. L. (Org.). Gerência em ação: singularidades e dilemas do trabalho gerencial. Rio de janeiro: FGV, 2005.

DOLAN, S. L. Estresse, auto-estima, saúde e trabalho. Rio de Janeiro: Qualitymark, 2006.

FRENCH, W. L. Organization development: theory, practice, research. Texas: Business Publications, 1983.

GONÇALVES, C. A.; MEIRELLES, A. M. Projetos e relatórios de pesquisa em administração. São Paulo: Atlas, 2004.

ISMA - International Stress Management Association. Estocolmo - Suécia, 2003. (Bulletin).

ISMA - International Stress Management Association. Estocolmo - Brasil, 2004. (Relatório de Pesquisa)

KARASEK, R. et al. The job content questionnaire (JCQ): a instrument for internationally comparative assessments of psychosocial job characteristics. Educational Publishing Foundation. Journal of Occupational Health Psychology. v. 3, n. 4, p. 322-355, 1998.

KARASEK, R.; TORRES T. Current issues relating to psychosocial job strain and cardiovascular. Journal of Occupational Health Psychology. v. 1, n. 1, p. 9-26, 1996.

KLIKSBERG, B. A gerência no final do século XX. Revista de Administração Pública, v. 27, n. 2, p. 183-201, abr./jun. 1993. 
LAZARUS, R. S. Personalidade e adaptação. 3 ed. Álvaro Cabral (trad.). Rio de Janeiro: Zahar, 1974.

LEONTIEV, A. O desenvolvimento do psiquismo. Lisboa: Horizonte Universitário, 1978.

LEMOS, C. Inovação na era do conhecimento. Ciência, Tecnologia e Sociedade, n. 8, maio/2000.

LIMONGI-FRANÇA, A. C.; RODRIGUES, A. L. Stress e trabalho: uma abordagem psicossomática. 4. ed. São Paulo: Atlas, 2005.

LIPP, M. E. N. Stress e o turbilhão da raiva. São Paulo: Caso do Psicólogo, 2005.

LEVI, L. O guia da comissão européia sobre stress relacionado ao trabalho e iniciativas relacionadas: das palavras à ação. In: ROSSI, A. M., PERREWÉ, P. L., SAUTER, S. L. (organizadores). Stress e qualidade de vida no trabalho: perspectivas atuais da saúde ocupacional. São Paulo: Atlas, 2005.

LHUILHIER, D.; MIGNÉE, C.; RAIX, A. Approches Psychosociales du stress. In : XXI Journées Nationales, Thème 3: Santé Mentale em Milieu de Travail, Rouen, p. 257-261, 1990.

MAFFIA, L. N. Estresse ocupacional em gestores: estudo nas secretarias de estado de Minas Gerais. Dissertação (Mestrado em Administração). Programa de Pós-Graduação da Universidade Federal de Minas Gerais - UFMG, Belo Horizonte, MG, 2013.

MELO, M. C. O. L. Impactos da prática organizacional do novo capitalismo na função gerencial. In: III Congresso Latino-americano de Sociologia del Trabajo. Anais... Buenos Aires, 2000.

MORAIS, L. F. R.; MARQUES, A. L.; ZILLE, L. P. Qualidade de vida e estresse organizacional: um estudo comparativo em profissões de risco. (Relatório de Pesquisa). Belo Horizonte: CEPEAD/UFMG, 1998.

NASCIMENTO, A. B. et al. Os efeitos do estresse sobre o tempo de reação e habilidade de resolução rápida de problemas. In: I Encontro Interno de Psicobiologia, 1998, Londrina. Anais... Londrina, p. 12-12, 1998.

PERREWÉ, P. L. As conseqüências do humor deprimido no local de trabalho: uma perspectiva teórica. In: ROSSI, A. M.; PERREWÉ, P. L.; SAUTER, S. L. (organizadores). Stress e qualidade de vida no trabalho: perspectivas atuais da saúde ocupacional. São Paulo: Atlas, 2005.

ROSSI, A. M. Estressores ocupacionais e diferenças de gênero. In: ROSSI, A. M.; PERREWÉ, P. L.; SAUTER, S. L. (Org.). Stress e qualidade de vida no trabalho: perspectivas atuais da saúde ocupacional. São Paulo: Atlas, 2005.

ROSSI, A. M.; PERREWÉ, P. L.; SAUTER, S. L. (Org.). Stress e qualidade de vida no trabalho: perspectivas atuais da saúde ocupacional. São Paulo: Atlas, 2005.

ROSSI, L. G. A. Novas demandas para a função gerencial e os gerentes de pequenas indústrias na região metropolitana de Belo Horizonte. Dissertação (Mestrado em Administração). Programa de Pós-Graduação da Universidade Federal de Minas Gerais - UFMG, Belo Horizonte, MG, 2001.

ROULEAU. L. Gerentes e repertórios de identidade em contexto de reestruturação organizacional. In: Gerência em ação: singularidades e dilemas do trabalho gerencial. Rio de Janeiro: FGV, 2005.

SANT'ANNA, A. S.; KILIMNIK, Z. M. Relações entre qualidade de vida no trabalho e estresse ocupacional: perspectivas teóricas. In: SANT'ANNA, A. S.; KILIMNIK, Z. M. (Orgs.). Qualidade de vida no trabalho: abordagens e fundamentos. Belo Horizonte: Elsevier/Fundação Dom Cabral, 2011.

SAUTER, S. L.; MURPHY, L. R. Abordagens à prevenção do estresse no trabalho dos Estados Unidos. In: ROSSI, A. M.; PERREWÉ, P. L.; SAUTER, S. L. (Org.). Stress e qualidade de vida no trabalho: perspectivas atuais da saúde ocupacional. São Paulo: Atlas, 2005.

SELYE, H. A syndrome produced by diverse nocuous agents. Nature, v. 138, n. 32, 1936.

SELYE, H. The stress of life. Toronto: McGraw-Hill, 1956.

SELYE, H. Stress without distress. New York: NAL Penguin Inc, 1974.

VELOSO, H. M. O que o coração sente mesmo quando os olhos não vêem: levantamento e análise dos agentes estressantes do trabalho bancário. Belo Horizonte: CEPEAD/UFMG, 2000. 
VERGARA, S. C. Projetos e relatórios de pesquisa em administração. São Paulo: Atlas, 2007.

ZILLE, L. P. Novas perspectivas para a abordagem do estresse ocupacional em gerentes: estudo em organizações brasileiras de setores diversos. 2005. Tese (Doutorado em Administração). Programa de Pós-Graduação da Universidade Federal de Minas Gerais - UFMG, Belo Horizonte, MG, 2005.

ZILLE, L. P.; BRAGA, C. D. Estresse ocupacional e as principais fontes de tensão no trabalho: impactos na produtividade de gestores do setor de energia elétrica brasileiro. In: XXXIV Associação Nacional De Pós-Graduação E Pesquisa Em Administração, 2010, Rio de Janeiro. Anais... Rio de Janeiro, 2010.

ZILLE, L. P.; BRAGA, C. D; ZILLE, G. P. Estresse ocupacional: como os gestores brasileiros estão respondendo às transformações gerenciais. In: SANT'ANNA, A. S.; KILIMNIK, Z. M. Qualidade de vida no trabalho: fundamentos e abordagens. Rio de Janeiro: Elsevier, $2011 \mathrm{a}$.

ZILLE, L. P. O estresse e a função gerencial: buscando as interfaces da realidade brasileira e portuguesa. In: BARBOSA, A. C.; SILVA R. J. (Editores). Economia, gestão e saúde: as relações luso-brasileiras em perspectiva. Lisboa: Edições Colibri, 2011b.

NOTA

1 A dislipidemia é o aumento anormal da taxa de lipídios no sangue. Representa um importante fator de risco para o desenvolvimento de lesões ateroscleróticas que podem causar a obstrução total do fluxo sanguíneo e apresenta altos índices de mortalidade. As dislipidemias podem ocorrer por causa do aumento das triglicérides (TGs) - (hipertrigliceridemia isolada), aumento do colesterol (hipercolesterolemia isolada) ou por uma combinação das duas (dislipidemia mista). Pode ainda ser causada pela redução do HDL ou aumento dos TGs ou LDL-C. Fonte: HTTP://www.roche.com.br (Acesso em: 19/02/2008).

(*) Pesquisa Apoiada pela FAPEMIG. 